\title{
Spin Coulomb drag in the two-dimensional electron liquid
}

\author{
Irene D'Amico \\ Istituto Nazionale per la Fisica della Materia (INFM) and Institute for Scientific Interchange, \\ via Settimio Severo 65, I-10133 Torino, Italy \\ Giovanni Vignale \\ Department of Physics, University of Missouri, Columbia, Missouri 65211, USA
}

(Received 16 December 2001; revised manuscript received 1 March 2002; published 14 July 2003)

\begin{abstract}
We calculate the spin-drag transresistivity $\rho_{\uparrow \downarrow}(T)$ in a two-dimensional electron gas at temperature $T$ in the random-phase approximation. In the low-temperature regime we show that, at variance with the threedimensional low-temperature result $\left[\rho_{\uparrow \downarrow}(T) \sim T^{2}\right]$, the spin transresistivity of a two-dimensional spin unpolarized electron gas has the form $\rho_{\uparrow \downarrow}(T) \sim T^{2} \ln T$. In the spin-polarized case the familiar form $\rho_{\uparrow \downarrow}(T)=A T^{2}$ is recovered, but the constant of proportionality, $A$, diverges logarithmically as the spin-polarization tends to zero. In the high-temperature regime we obtain $\rho_{\uparrow \downarrow}(T)=-\left(\hbar / e^{2}\right)\left(\pi^{2} \mathrm{Ry}^{*} / k_{B} T\right)$ (where Ry* is the effective Rydberg energy) independent of the density. Again, this differs from the three-dimensional result, which has a logarithmic dependence on the density. Two important differences between the spin-drag transresistivity and the ordinary Coulomb-drag transresistivity are pointed out. (i) The $\ln T$ singularity at low temperature is smaller, in the Coulomb-drag case, by a factor $e^{-4 k_{F} d}$, where $k_{F}$ is the Fermi wave vector and $d$ is the separation between the layers. (ii) The collective mode contribution to the spin-drag transresistivity is negligible at all temperatures. Moreover, the spin-drag effect is, for comparable parameters, larger than the ordinary Coulomb-drag effect.
\end{abstract}

DOI: 10.1103/PhysRevB.68.045307

PACS number(s): 72.25.Dc, 72.10.-d

\section{INTRODUCTION}

The problem of transporting an electronic spin polarization from a place to another by means of an electrical current has attracted tremendous interest in recent years, both in theoretical and in experimental circles. ${ }^{1}$ A particularly challenging problem is that of injecting spin-polarized electrons from a ferromagnet into an ordinary nonmagnetic semiconductor such as GaAs. This is by no means easy, due to the large impedance mismatch between the ferromagnetic injector and the semiconductor. ${ }^{2}$ However, the discovery that GaAs, when doped with magnetic Mn impurities, becomes ferromagnetic with a Curie temperature $T_{c}$ as large as $110 \mathrm{~K}$ (Ref. 3) has raised hopes of realizing useful all-semiconductor spinelectronic devices in a near future.

In dealing with spin-polarized currents one must be prepared to treat a situation in which electrons of opposite spin orientations travel, on the average, with different speeds. For example, the up-spin electrons injected from a ferromagnet into a semiconductor might be drifting in one direction, while the down-spin electrons remain, on the average, stationary. Whenever such a situation occurs, the existence of Coulomb correlation between up spins and down spins becomes important, because it is a source of friction between the two components. In our previous works ${ }^{4-6}$ we examined the effect of up-down spin correlations on spin polarized transport in a three-dimensional electron gas, and we introduced the concept of spin Coulomb drag (SCD): ${ }^{4}$ due to Coulomb scattering, momentum is transferred between the spin populations, tending to equalize their average drift velocities. This process represents an actual drag exerted by the slower population on the faster and, in the absence of an external driving field, leads to a decay of the spin current even in the absence of extrinsic impurities. ${ }^{7}$ Later, it was shown that the SCD also limits the diffusion of a spin packet in a doped semiconductor, ${ }^{5}$ adding to the conventional effect of impurity scattering.

The quantitative measure of the SCD is the spin-drag transresistivity $\rho_{\uparrow \downarrow}(T)$, which is defined as the ratio of the gradient of electrochemical potential for up-spin electron $E_{\uparrow}$ to the current of down-spin electrons $j_{\downarrow}$ when the current of up-spin electrons is zero:

$$
E_{\uparrow}=\rho_{\uparrow \downarrow}(T) j_{\downarrow} \quad\left(j_{\uparrow}=0\right) .
$$

In Ref. 4 we presented a calculation of $\rho_{\uparrow \downarrow}(T)$ in a pure three-dimensional electron gas in the random-phase approximation (RPA). The calculation is analogous to that of the more familiar Coulomb-drag effect, ${ }^{8}$ but, as we will show below, some important differences exist between the two effects.

The ordinary Coulomb-drag (CD) effect involves two distinct quasi-two-dimensional electron layers that are spatially separated by an insulating barrier. By contrast, in the SCD, we are dealing with a single layer in which the two carrier populations (distinguishable by the different spin) share the same space and interact with the same impurities. ${ }^{9}$ It follows that the SCD can be either a two-dimensional (2D) or a fully 3D effect, depending on the dimension of the space in which the electrons move. We have already shown that the 3D spin transresistivity should be measurable in metals ${ }^{4}$ and becomes larger in semiconductors, ${ }^{5,6}$ where it can be of the same order of magnitude as the usual Drude resistivity. In this paper we concentrate on the 2D case. Due to the absence of spatial separation between the two spin populations, the bare interaction between parallel and antiparallel spin electrons coin- 
cides with the interaction between parallel spin electrons, and its Fourier transform is simply $v(q)=2 \pi e^{2} / q \epsilon$, where $q$ is the wave vector in the plane and $\epsilon$ is the appropriate lowfrequency dielectric constant. ${ }^{10}$ By contrast, in the standard $\mathrm{CD}$ case, the bare interaction between electrons in the two layers has the 2D Fourier transform $v_{12}(q)$ $=2 \pi e^{2} e^{-q d} / q \epsilon$, where $d$ is the distance between the layers. This "innocent" difference has two important consequences. First, there is no acoustic-plasmon-mediated interaction in SCD, whereas the effect of acoustic plasmons on the temperature dependence of the $\mathrm{CD}$ in separate layers is quite marked. ${ }^{11}$ Second, the effective interaction between up and down spins in SCD remains sizable up to the values of $q$ comparable to the Fermi momentum: the presence of a significant interaction strength at $q=2 k_{F}$ is responsible for a characteristic logarithmic singularity in the low-temperature transresistivity.

We provide analytical expressions for $\rho_{\uparrow \downarrow}$ in both the lowand high-temperature limits. At low temperature we derive analytically the $T^{2} \ln T$ singularity; at high temperature, we show that $\rho_{\uparrow \downarrow}$ becomes density independent. Both these limits are significant for realistic metallic and semiconductor parameters, respectively. We also draw a comparison between the spin Coulomb drag and the ordinary Coulomb drag. It is hoped that the theoretical results presented in this paper will stimulate experimental work aimed at a direct observation of the SCD and a comparison between SCD and $\mathrm{CD}^{12}$

\section{SPIN TRANSRESISTIVITY IN THE 2D ELECTRON LIQUID}

To leading order in the strength of the Coulomb interaction, ${ }^{13}$ the spin-drag transresistivity is given by Eq. (18) of Ref. 4, which, in the static case and after few simple rearrangements, can be written as

$$
\begin{aligned}
\rho_{\uparrow \downarrow}(T)= & -\frac{\hbar}{e^{2}} \frac{\hbar}{n_{\uparrow} n_{\downarrow} k_{B} T} \frac{C_{D}}{(2 \pi)^{D+1}} \int_{0}^{\infty} d q \frac{q^{D+1}}{D} \\
& \times \int_{0}^{\infty} d \omega \frac{\operatorname{Im} Q_{0 \uparrow}(q, \omega) \operatorname{Im} Q_{0 \downarrow}(q, \omega)}{|\epsilon(q, \omega)|^{2} \sinh ^{2}\left(\hbar \omega / 2 k_{B} T\right)} .
\end{aligned}
$$

Here $k_{B}$ is the Boltzmann constant, $D$ is the number of spatial dimensions, and $C_{D}=1,2 \pi, 4 \pi$ for $D=1,2,3$, respectively. The dimensionless function $Q_{0 \sigma}(q, \omega)$ is defined as

$$
Q_{0 \sigma}(q, \omega) \equiv v(q) \chi_{0 \sigma}(q, \omega),
$$

where $\chi_{0 \sigma}(q, \omega)$ is the noninteracting density-density response function (the "Lindhard function") for spin $\sigma=\uparrow$ or $\downarrow$ and $v(q)$ is the Fourier transform of the Coulomb interaction. $\epsilon(q, \omega)=1-Q_{0 \uparrow}(q, \omega)-Q_{0 \downarrow}(q, \omega)$ is the RPA dielectric function of the electron liquid, and $n_{\uparrow}$ and $n_{\downarrow}$ are the densities of up- and down-spin electrons. In both two and three dimensions $\operatorname{Im} \chi_{0 \sigma}$ can be expressed analytically. In particular, in two dimensions, starting from the familiar expression

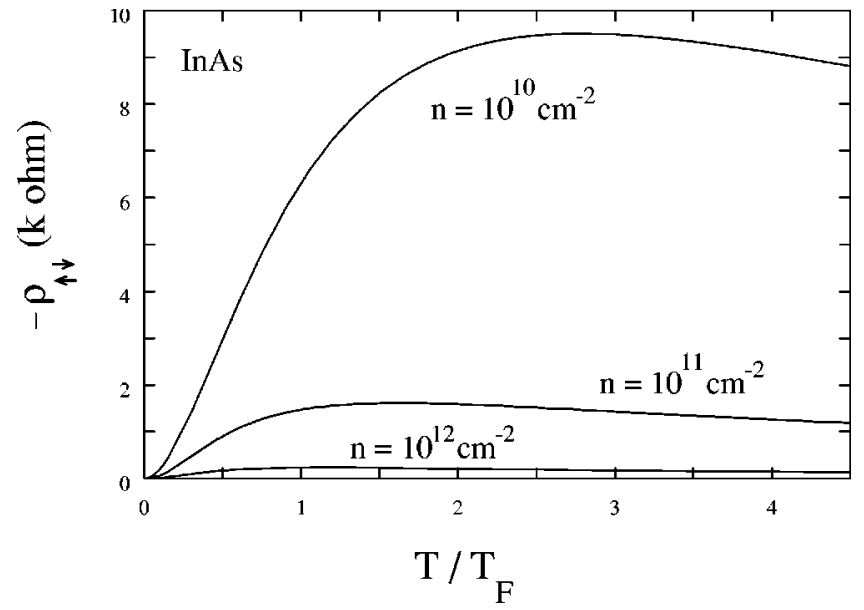

FIG. 1. Spin transresistivity $\rho_{\uparrow \downarrow}$ as a function of temperature (rescaled by $\left.T_{F}\right)$ for InAs parameters $\left(m^{*}=0.026 m_{e}, \epsilon=13.6\right)$. Each curve corresponds to a different density as labeled.

$$
\begin{aligned}
\operatorname{Im} \chi_{0 \sigma}(q, \omega)= & -\pi \int \frac{d^{2} k}{(2 \pi)^{2}} n_{k} \\
& \times\left\{\delta\left(\hbar \omega+E_{k}-E_{k-q}\right)-\delta\left(\hbar \omega-E_{k}+E_{k-q}\right)\right\},
\end{aligned}
$$

with $E_{k}=\hbar^{2} k^{2} / 2 m^{*}$ and $n_{k}=1 /\left(\exp \left[\left(E_{k}-\mu\right) / k_{B} T\right]+1\right)\left(m^{*}\right.$ is the effective mass), we obtain

$$
\begin{aligned}
\operatorname{Im} Q_{0 \sigma}(q, \omega)= & -\frac{k_{F \sigma} \sqrt{\bar{T}_{\sigma}}}{q^{2} a^{*}} \\
& \times\left[F\left(e^{\left(\nu_{\sigma-}^{2}-\bar{\mu}_{\sigma}\right) / \bar{T}_{\sigma}}\right)-F\left(e^{\left(\nu_{\sigma+}^{2}-\bar{\mu}_{\sigma}\right) / \bar{T}_{\sigma}}\right)\right] .
\end{aligned}
$$

Here $a^{*}=\hbar^{2} \epsilon / m e^{2}$ is the effective Bohr radius, $k_{F \sigma}$ is the Fermi wave vector of $\sigma$-spin electrons, $\bar{T}_{\sigma}=k_{B} T / E_{F \sigma}$ and $\bar{\mu}_{\sigma}=\mu_{\sigma} / E_{F \sigma}$ are the temperature and chemical potential of $\sigma$-spin electrons expressed in units of the $\sigma$-spin Fermi energy $E_{F \sigma}$, and

$$
\nu_{\sigma \pm} \equiv \frac{\omega}{q v_{F \sigma}} \pm \frac{q}{2 k_{F \sigma}},
$$

where $v_{F \sigma}=\hbar k_{F \sigma} / m^{*}$ is the $\sigma$-spin Fermi velocity.

The function $F$ in Eq. (5) is defined as

$$
F(z)=\int_{0}^{\infty} \frac{d x}{z e^{x^{2}}+1} .
$$

In Fig. 1 we present the behavior of $\rho_{\uparrow \downarrow}(T)$ calculated from Eq. (2) and $D=2$ for three different values of the carrier density with the values of the effective mass and dielectric constant appropriate for InAs $\left(m^{*}=0.026 m_{e}, m_{e}\right.$ being the free electron mass, and $\epsilon=13.6)$. As pointed out in Refs. 4,6 , the spin transresistivity increases with decreasing densities. As a function of temperature, $\rho_{\uparrow \downarrow}$ vanishes at $T=0$, reaches a maximum about the Fermi temperature $T_{F}$, and decreases for large $T$. As shown in Fig. 1, the overall scale of the effect, at its largest, is set by $\hbar / e^{2} \simeq 4.1 \mathrm{k} \Omega$. This is quite a large value and should definitely be observable. 

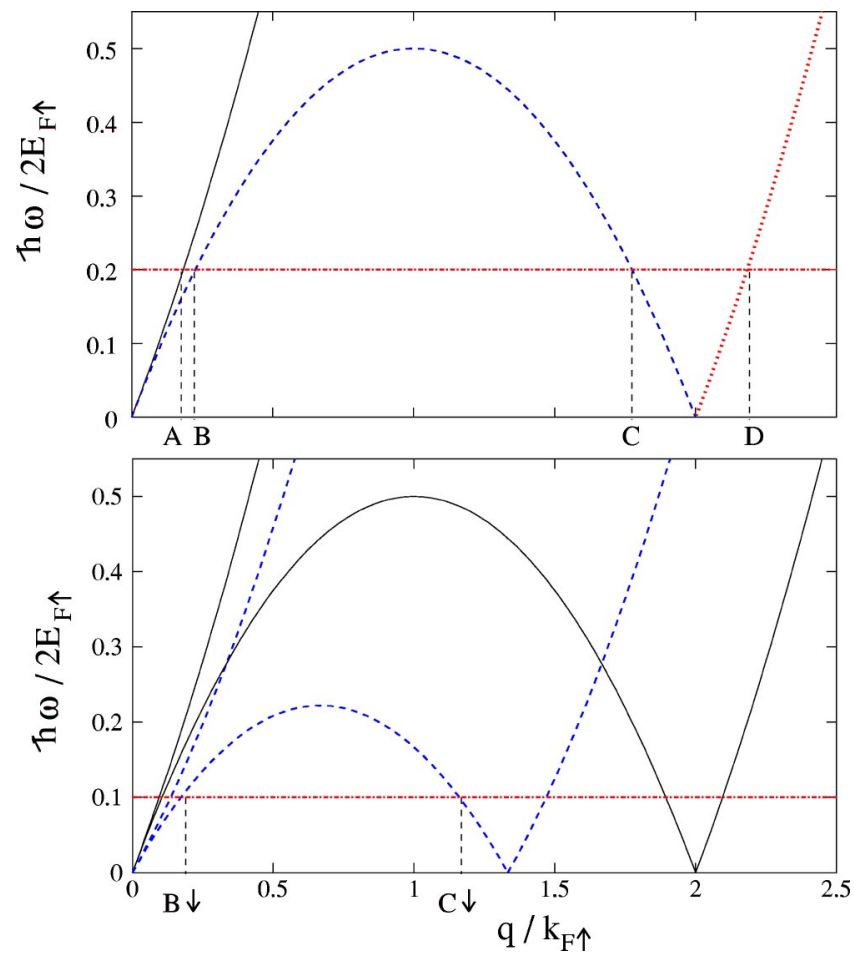

FIG. 2. Parameter space for the existence of a nonzero $\operatorname{Im} Q_{0 \sigma}(q, \omega)$ at $T=0$. (Upper panel) Paramagnetic case $\left(k_{F \uparrow} / k_{F \downarrow}\right.$ $=1)$. The three curves correspond to the set of points in which one or both of the arguments of the Heaviside functions in Eq. (8) are equal to zero: $\left(q / k_{F}\right)^{2} / 2+q / k_{F}$ (solid curve), $-\left(q / k_{F}\right)^{2} / 2+q / k_{F}$ (dashed curve), and $\left(q / k_{F}\right)^{2} / 2-q / k_{F}$ (dotted curve). $\operatorname{Im} Q_{0 \sigma}(q, \omega)$ is different from zero in the region between the solid and the dotted curve. The points labeled by $A-D$ correspond, respectively, to $q_{A \sigma}(\omega)-q_{D \sigma}(\omega)$ for $\hbar \omega / 2 E_{F \sigma}=0.2$. (Lower panel) Spin-polarized case $\left(k_{F \uparrow} / k_{F \downarrow}=1.5\right)$. The solid curve defines the parameter space corresponding to $\operatorname{Im} Q_{0 \uparrow}(q, \omega)$, the dashed curve the one related to $\operatorname{Im} Q_{0 \downarrow}(q, \omega)$. The points labeled as $B \downarrow$ and $C \downarrow$ correspond to $q_{B \downarrow}$ and $q_{C \downarrow}$, respectively, and delimit the interval relevant to the calculation of Eq. (14).

We now derive and discuss the degenerate and nondegenerate limits of Eq. (2).

\section{THE DEGENERATE LIMIT}

In the limit $T \ll T_{F}$ the calculation of $\rho_{\uparrow \downarrow}(T)$ can be carried out analytically. The temperature dependence of $\rho_{\uparrow \downarrow}$, in this regime, is entirely determined by the denominator $\sinh ^{2}\left(\hbar \omega / 2 k_{B} T\right)$, which restricts the integral in Eq. (2) to frequencies of the order of $k_{B} T$. We can therefore neglect the temperature dependence of $\operatorname{Im} Q_{0 \sigma}$ and replace this function by its zero-temperature limit

$$
\begin{aligned}
\operatorname{Im} Q_{0 \sigma}(q, \omega)= & -\frac{k_{F \sigma}}{q^{2} a^{*}}\left[\Theta\left(1-\nu_{\sigma^{-}}^{2}\right) \sqrt{1-\nu_{\sigma^{-}}^{2}}\right. \\
& \left.-\Theta\left(1-\nu_{\sigma+}^{2}\right) \sqrt{1-\nu_{\sigma+}^{2}}\right] .
\end{aligned}
$$

The behavior of $\operatorname{Im} Q_{0 \sigma}(q, \omega)$ for small $\omega$ depends crucially on the value of $q$. As shown in the upper panel of Fig. 2, there are three distinct regions in which $\operatorname{Im} Q_{0 \sigma}(q, \omega)$ is different from zero:

$$
\begin{gathered}
q_{A \sigma} \equiv k_{F \sigma}\left[-1+\sqrt{1+\frac{2 \omega}{k_{F \sigma} v_{F \sigma}}}\right] \\
<q<k_{F \sigma}\left[1-\sqrt{1-\frac{2 \omega}{k_{F \sigma} v_{F \sigma}}}\right] \equiv q_{B \sigma}, \\
\operatorname{Im} Q_{0 \sigma}(q, \omega) \simeq-\frac{k_{F \sigma}}{q^{2} a^{*}} \sqrt{\frac{\omega}{k_{F \sigma} v_{F \sigma}}}
\end{gathered}
$$

$$
\begin{aligned}
q_{B \sigma} & \equiv k_{F \sigma}\left[1-\sqrt{1-\frac{2 \omega}{k_{F \sigma} v_{F \sigma}}}\right] \\
& <q<k_{F \sigma}\left[1+\sqrt{1-\frac{2 \omega}{k_{F \sigma} v_{F \sigma}}}\right] \equiv q_{C \sigma}
\end{aligned}
$$

$$
\operatorname{Im} Q_{0 \sigma}(q, \omega) \simeq-\frac{k_{F \sigma}}{q^{2} a^{*}} \frac{\omega /\left(k_{F \sigma} v_{F \sigma}\right)}{\sqrt{1-\left(q / 2 k_{F \sigma}\right)^{2}}}
$$

$$
\begin{aligned}
q_{C \sigma} & \equiv k_{F \sigma}\left[1+\sqrt{1-\frac{2 \omega}{k_{F \sigma} v_{F \sigma}}}\right] \\
& <q<k_{F \sigma}\left[1+\sqrt{1+\frac{2 \omega}{k_{F \sigma} v_{F \sigma}}}\right] \equiv q_{D \sigma},
\end{aligned}
$$

$$
\operatorname{Im} Q_{0 \sigma}(q, \omega) \simeq-\frac{k_{F \sigma}}{q^{2} a^{*}} \sqrt{\frac{\omega}{k_{F \sigma} v_{F \sigma}}+1-\frac{q^{2}}{4 k_{F \sigma}^{2}}} .
$$

Let us first consider the paramagnetic case $n_{\uparrow}=n_{\downarrow}, k_{F \uparrow}$ $=k_{F \downarrow}$ etc. $\operatorname{In}$ region (I) the product $\operatorname{Im} Q_{0 \uparrow} \operatorname{Im} Q_{0 \downarrow}$ is proportional to $\omega$, while the integration region in the $q$ space is a shell of thickness proportional to $\omega^{2}$. The net $q$ dependence of the integrand [taking into account the fact that $\epsilon(q, 0)$ $\left.\simeq 1+2 /\left(q a^{*}\right),|\epsilon(q, 0)|^{2} \sim 1 /\left(q a^{*}\right)^{2}\right]$ is $\propto q$, giving an extra factor $\omega / v_{F}$. Since $\hbar \omega \sim k_{B} T$, the contribution of this region to $\rho_{\uparrow \downarrow}$ is at least of order $T^{4}$ and will be disregarded hereafter.

In region (II) the product $\operatorname{Im} Q_{0 \uparrow} \operatorname{Im} Q_{0 \downarrow}$ is proportional to $\omega^{2}$, while the integration region in the $q$ space is a shell of thickness $\sim 2 k_{F}$. This would give a contribution of order $T^{2}$ (as in 3D) were it not for the square root divergence of $\operatorname{Im} Q_{0 \sigma}$ when $q$ approaches $2 k_{F}$ [see Eq. (10)]. Due to the "piling-up" of two square-root singularities, one gets an additional $\ln (\omega)$ from the upper limit $\left(q \sim 2 k_{F}\right)$ of the integration interval. Thus the total contribution of this region to $\rho_{\uparrow \downarrow}$ is of order $T^{2} \ln T$. Notice that this logarithmic contribution would be severely suppressed in the case of the ordinary 
Coulomb drag, since the Fourier transform of the Coulomb interaction between electrons in different layers decreases, with increasing $q$, as $e^{-q d}$.

Finally, in region (III), the product $\operatorname{Im} Q_{0 \uparrow} \operatorname{Im} Q_{0 \downarrow}$ is again proportional to $\omega$, but the integration region in the $q$ space is now a shell of thickness $\sim \omega / v_{F}$ centered about $2 k_{F}$. The contribution of this region is therefore of order $T^{2}$.

Combining the contributions of regions (II) and (III) together, after some calculations, we arrive at the following expression:

$$
\begin{aligned}
\rho_{\uparrow \downarrow}(T) \stackrel{T \ll T_{F}}{\approx} & -\frac{\hbar}{e^{2}} \frac{2 \pi^{2} r_{s}^{2}}{3\left(\sqrt{2}+r_{s}\right)^{2}}\left(\frac{k_{B} T}{E_{F}}\right)^{2}\left[f\left(\frac{r_{s}}{\sqrt{2}}\right)-\frac{1}{2} \ln \left(\frac{k_{B} T}{E_{F}}\right)\right. \\
& \left.+\ln 2+1-\frac{3}{\pi^{2}} \int_{0}^{\infty} \frac{y^{2} \ln y}{\sinh ^{2} y} d y\right],
\end{aligned}
$$

where $r_{s}=1 / \sqrt{\pi n} a^{*}$ is the dimensionless Wigner-Seitz radius, $\int_{0}^{\infty}\left(y^{2} \ln y / \sinh ^{2} y\right) d y=-0.55981$, and the function $f(x)$ is defined as

$$
f(x):=\frac{1}{x-1}\left[1+\frac{x^{2}+1}{x-1} \ln \left(\frac{1+x}{2 x}\right)\right] .
$$

Thus, in the paramagnetic case the spin transresistivity behaves as $T^{2} \ln T$ rather than $T^{2}$.

This is no longer true if the electron gas is spin polarized. Let us assume $n_{\uparrow}>n_{\downarrow}$ so that $k_{F \uparrow}>k_{F \downarrow}$. For a finite polarization and at sufficiently low temperatures such that $k_{B} T$ $\ll E_{F \uparrow}$ and $k_{B} T \ll E_{F \downarrow}, \operatorname{Im} Q_{0 \sigma}(q, \omega)$ can be approximated with its zero-temperature expression and the region of integration in $q$ coincides with the region in which $\operatorname{Im} Q_{0 \downarrow}(q, \omega)$ differs from zero (see lower panel of Fig. 2). As in the paramagnetic case, it is then possible to divide the $q$-integral into regions in which $\operatorname{Im} Q_{0 \sigma}$ takes values given by Eqs. (9)-(11). At the upper limit of region (II) for down spins [Eq. (10)], $q=2 k_{F \downarrow}-\omega / v_{F \downarrow}, \quad \operatorname{Im} Q_{0 \downarrow}(q, \omega)$ diverges as $1 / \sqrt{1-\left(q / 2 k_{F \downarrow}\right)^{2}}$. However, $\operatorname{Im} Q_{0 \uparrow}(q, \omega)$ remains regular. Therefore, the logarithmic divergence leading to the $\ln T$ singularity in the transresistivity is cut off.

Our final result takes the form

$$
\rho_{\uparrow \downarrow}(T) \stackrel{T \ll T_{F}}{\approx}-\frac{\hbar}{e^{2}} \frac{\pi^{2} r_{s}^{2}}{3\left(1-\xi^{2}\right)^{3 / 2}}\left(\frac{k_{B} T}{E_{F}}\right)^{2} g\left(r_{s}, \xi\right),
$$

where function $g$ is defined as

$$
g\left(r_{s}, \xi\right)=\int_{0}^{1} d x \frac{x}{\left[x+\frac{r_{s}}{\sqrt{2(1-\xi)}}\right]^{2} \sqrt{(1-\alpha x)(1-x)}}
$$

$\xi=\left(n_{\uparrow}-n_{\downarrow}\right) /\left(n_{\uparrow}+n_{\downarrow}\right)$ is the degree of spin polarization and $\alpha \equiv \sqrt{1-\xi} / \sqrt{1+\xi}$. Notice that $g\left(r_{s}, \xi\right)$ diverges logarithmically for $\xi \rightarrow 0$, in agreement with our previous findings.

Figure 3 focuses on the paramagnetic degenerate regime: it presents a comparison between the numerical evaluation of $\rho_{\uparrow \downarrow}$ from Eq. (2) (curve labeled $C$ ) and its analytical approximation Eq. (12) (curve labeled $B$ ). The curve labeled as

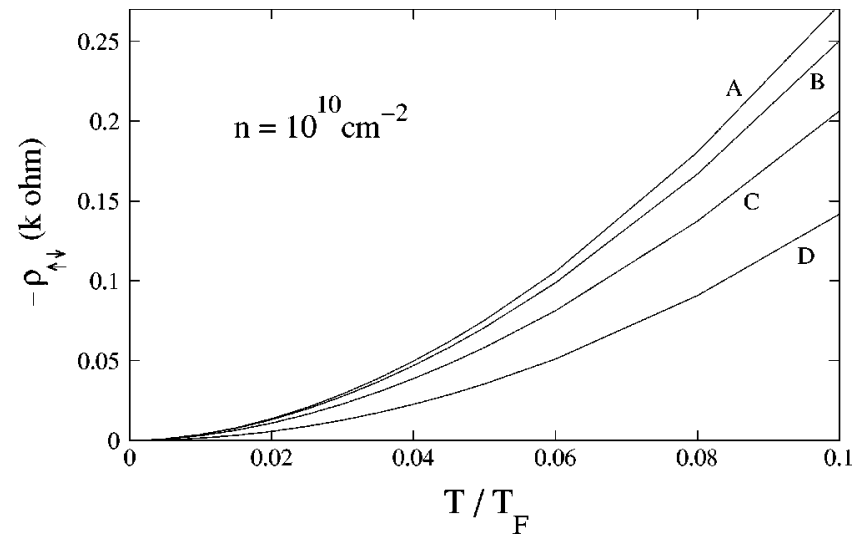

FIG. 3. Degenerate regime: Comparison between $\rho_{\uparrow \downarrow}$ (curve labeled $C$ ), its analytical approximation Eq. (12) (curve labeled $B$ ), the approximation obtained using the zero-temperature form of $\operatorname{Im} Q_{0 \sigma}(q, \omega)$ in Eq. (2) (curve labeled $A$ ), and the analytic one obtained by neglecting the $T^{2} \ln T$ term in Eq. (12) (curve labeled $D$ ). The curves are plotted vs temperature (rescaled by $T_{F}$ ) for $n$ $=10^{10} \mathrm{~cm}^{-2}$ and InAs parameters.

$D$ is obtained neglecting the $T^{2} \ln T$ term in Eq. (12): by comparing the $D$ curve with the $B$ curve, the importance of the $T^{2} \ln T$ correction becomes evident: without it, the spin drag is strongly underestimated. The $A$ curve represents the result obtained using in Eq. (2) the zero-temperature limit for $\operatorname{Im} Q_{0 \sigma}(q, \omega)$. As expected, Fig. 3 shows that in this range of temperatures, the analytical approximation ( $B$ curve) is very close to such a result.

\section{THE NONDEGENERATE LIMIT}

In the nondegenerate limit $k_{B} T \gg E_{F}$, the dimensionless chemical potential $\bar{\mu}_{\sigma}$ of Eq. (5) is given by the classical formula $\bar{\mu}_{\sigma}=\bar{T}_{\sigma} \ln \left(\hbar^{2} 2 \pi n_{\sigma} / m^{*} k_{B} T\right)$. Since $-\bar{\mu}_{\sigma} / \bar{T}_{\sigma} \rightarrow \infty$ we can replace the function $F(z)$ [Eq. (7)] by its limiting form $F(z) \rightarrow \sqrt{\pi} / 2 z$ for $z \rightarrow \infty$.

This leads to the result

$$
\begin{aligned}
\operatorname{Im} Q_{0 \sigma}(q, \omega) \stackrel{T \gg T_{F}}{\approx} & \frac{1}{a^{*}} \frac{E_{F \sigma}}{k_{B} T} \sqrt{\frac{m^{*} \pi}{2 k_{B} T}} \\
& \times \frac{\omega}{q^{2}} e^{-m^{*} \omega^{2} / 2 k_{B} T q^{2}} e^{-\hbar^{2} q^{2} / 8 m^{*} k_{B} T},
\end{aligned}
$$

identical to the 3D case. The Gaussian factor $e^{-\hbar^{2} q^{2} / 8 m^{*} k_{B} T}$ on the right-hand side of this equation tends to 1 in the classical limit (i.e., for $\hbar \rightarrow 0$ ) for any finite wave vector. However, we cannot set $\hbar=0$, because the integral extends to arbitrarily large wave vectors, and the classical approximation fails at large enough values of $q$. The Gaussian factor assures the convergence of the integral in Eq. (2) by cutting off the integral at values of $q$ such that $\hbar^{2} q^{2} / 2 m^{*} \sim k_{B} T$.

Using the classical expression for the dielectric constant $\epsilon(q, \omega) \approx 1+k_{D} / q$, with $k_{D}=2 \pi e^{2} n / \epsilon k_{B} T$, after simple manipulations, we finally obtain 


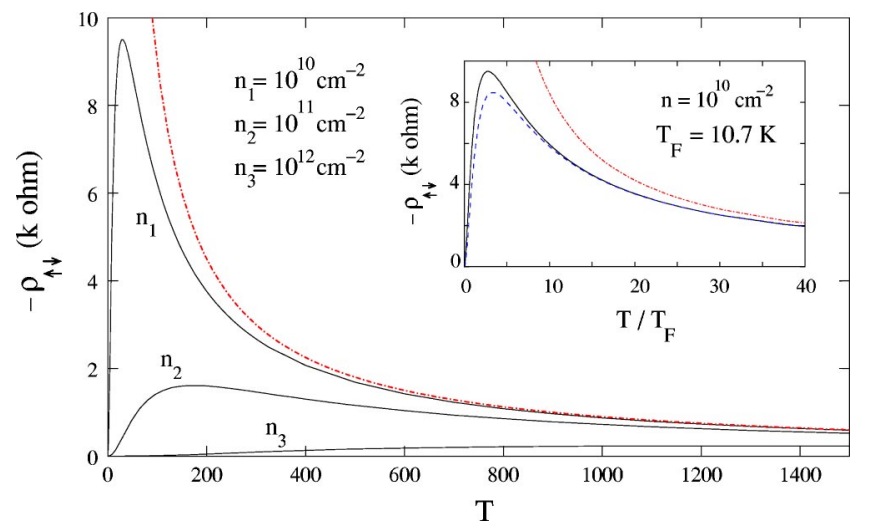

FIG. 4. Nondegenerate regime: Comparison between $\rho_{\uparrow \downarrow}$ (solid line) and its universal limiting behavior (dash-dotted line) Eq. (18) vs temperature for different carrier densities and InAs parameters. Inset: Comparison between $\rho_{\uparrow \downarrow}$ (solid line) and its approximations [Eq. (17) (dashed line) and Eq. (18) (dashed-dotted line)]. The curves are calculated for $n=10^{10} \mathrm{~cm}^{-2}$ and plotted vs temperature (rescaled by the Fermi temperature).

$$
\begin{aligned}
\rho_{\uparrow \downarrow}(T) & \stackrel{T \gg T_{F}}{\approx}-\frac{\hbar}{e^{2}} \pi^{3 / 2} \frac{\mathrm{Ry}^{*}}{k_{B} T} \int_{0}^{\infty} d x \frac{\sqrt{x} e^{-x}}{(\sqrt{x}+\lambda)^{2}} \\
& >-0 \\
& \approx-\frac{\hbar}{e^{2}} \pi^{2} \frac{\mathrm{Ry}^{*}}{k_{B} T},
\end{aligned}
$$

where $\lambda \equiv\left(2^{3 / 2} / r_{s}^{2}\right)\left(\mathrm{Ry}^{*} / k_{B} T\right)^{3 / 2}$ and $\mathrm{Ry}^{*}=m^{*} e^{4} / 2 \epsilon^{2} \hbar^{2}$ is the effective Rydberg.

Remarkably, the last result (corresponding to $k_{B} T \gg \mathrm{Ry} *$ or $r_{s} \gg 1$ ) is independent of the electronic density. It differs from the analogous result in three dimensions, which scales as $1 / T^{3 / 2} \ln (T)$ and has a weak (logarithmic) dependence on the electronic density. ${ }^{6}$

The limiting form of the transresistivity Eq. (18) is shown in Fig. 4 (dashed-dot line) along with the results obtained from Eq. (2) for the same choice of carrier densities as in Fig. 1. We stress that this curve delimits the region of the $(n, T)$ plane occupied by the family of curves $\rho_{\uparrow \downarrow}(n, T)$ and is approached at lower and lower temperatures for decreasing densities (see Fig. 4). The inset of Fig. 4 shows the comparison between $\rho_{\uparrow \downarrow}$ calculated for $n=10^{10} \mathrm{~cm}^{-2}$ (solid line) and its limiting behaviors, Eqs. (17) (dashed line) and (18) (dashed-dot line). It is interesting to notice that, because of the low Fermi temperatures and at variance with the 3D case, in $2 \mathrm{D}$ the nondegenerate behavior of the spin transresistivity could be observed experimentally, since it corresponds, for reasonable densities, to temperatures as low as $100 \mathrm{~K}$ (see inset in Fig. 4). We also notice that the nondegenerate limit, Eq. (17), is in reality a good approximation even for temperatures as low as few $T_{F}$.

\section{DISCUSSION AND SUMMARY}

A few comments are now in order. As Eq. (12) shows, in the degenerate regime, the $2 \mathrm{D}$ spin transresistivity behaves as $T^{2}(A+B \ln T)$, in contrast to the 3D case in which $\rho_{\uparrow \downarrow}$ $\sim T^{2}$ (see Ref. 4). The additional logarithmic correction is

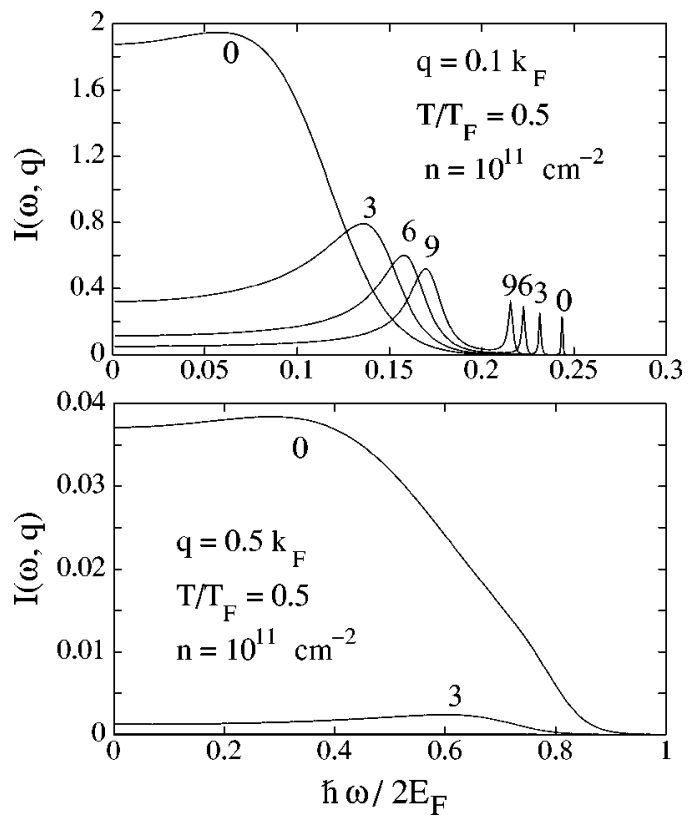

FIG. 5. Behavior of $\quad I(\omega, q) \equiv \exp$ $(-2 d q) \operatorname{Im} Q_{0 \uparrow}(q, \omega) \operatorname{Im} Q_{0 \downarrow}(q, \omega) / 4|\epsilon(q, \omega)|^{2} \sinh ^{2}\left(\hbar \omega / 2 k_{B} T\right)$ vs the rescaled frequency $\hbar \omega / 2 E_{F}$ for fixed $q$ and temperature but different values of $d\left(0 \leqslant d / k_{F} \leqslant 9\right)$. Upper panel: small- $q$ behavior ( $q$ $\left.=0.1 k_{F}\right)$. Each curve corresponds to a different value of $d / k_{F}$ as labeled. Lower panel: intermediate- $q$ behavior $\left(q=0.5 k_{F}\right)$. Curves are labeled with the corresponding value of $d / k_{F}$ (as in the upper panel).

due to the fast variation of $\operatorname{Im} Q_{0 \sigma}(q, \omega)$ for $q \approx 2 k_{F \sigma}$, so that the high- $q$ regime dominates the low-temperature behavior of $\rho_{\uparrow \downarrow}$. This correction is also present, in principle, in the more familiar Coulomb-drag transresistivity between two separate electron layers. However, in that case, due to the presence of the insulating barrier of thickness $d$, the Fourier transform of the interlayer Coulomb interaction is given by $2 \pi e^{2} e^{-q d} / \epsilon q$ and decays exponentially for large $q$. Thus, in $\mathrm{CD}$ the influence of the logarithmic term on the behavior of $\rho_{\uparrow \downarrow}$ in the degenerate regime is negligible.

The exponential decay of the interlayer interaction is responsible for another main difference between the Coulomb drag and the spin Coulomb drag. As can be seen in Ref. 11, for $T \lesssim T_{F}$ the transresistivity is dominated by the plasmon modes (especially the acoustic one), which enhance the contribution of small wave vectors. In the 2DSCD, however, the acoustic mode, in which the two electron populations oscillate out of phase, is absent. Figure 5 shows how the function $I(\omega, q) \equiv \exp (-2 d q) \operatorname{Im} Q_{0 \uparrow}(q, \omega) \operatorname{Im} Q_{0 \downarrow}(q, \omega) /\left[4|\epsilon(q, \omega)|^{2}\right.$ $\left.\times \sinh ^{2}\left(\hbar \omega / 2 k_{B} T\right)\right]$, behaves for small (upper panel) and intermediate (lower panel) values of $q$ in the paramagnetic regime. For $d=0, I(\omega, q)$ corresponds to the integrand of Eq. (2); in the figure, however, the interaction between the two spin populations is taken as $v_{\uparrow \downarrow}$ $=\left(2 \pi e^{2} / q\right) \exp (-q d)$ and the RPA dielectric function as $\epsilon(q, \omega)=\left[1-v(q) \chi_{0 \uparrow}(q, \omega)\right]\left[1-v(q) \chi_{0 \downarrow}(q, \omega)\right]$

$-v_{\uparrow \downarrow} \chi_{0 \uparrow}(q, \omega) \chi_{0 \downarrow}(q, \omega)$. Different curves correspond to different values of $d\left(0 \leqslant d / k_{F} \leqslant 9\right.$, as labeled). Let us first focus on the upper panel in which $q=0.1 k_{F}$ : for $d=0$ the 
main contribution to the integral comes from the single-pair excitation continuum. In this case the only possible collective mode [corresponding to the zeros of $\epsilon(q, \omega)]$ is the inphase optical mode, whose contribution (the spike labeled as 0 ) is in any case negligible. As the exponential factor in the interaction $v_{\uparrow \downarrow}$ is turned on (i.e., as the scattering rate between the two spin populations is decreased), the strength of the integrand is transferred from the single-pair excitation continuum to the collective modes and the out-of-phase acoustic plasmon appears (see the two maxima labeled with 9 , corresponding to $d=9 k_{F}$ ).

The lower panel of Fig. 5 presents $I(\omega, q)$ for $q$ $=0.5 k_{F}$ : already at this intermediate value, the contribution to $\rho_{\uparrow \downarrow}$ becomes negligible when $d>0$. In fact $I(\omega, q)$ scales as $v_{\uparrow \downarrow}^{2} \sim \exp (-2 q d)$.

We would like to emphasize a more general point: as Fig. 5 clearly shows, due to the absence of the exponential factor $e^{-q d}$ in the Fourier transform of the Coulomb potential, the drag effect in the SCD is definitely larger than in the ordinary CD. In other words, in SCD, the two electron populations can transfer momentum to one another more effectively.

The plasmon enhancement is absent in 3D as well. In this case, however, this is due to a combination of three different effects: (i) the 3D plasmon frequency $\omega_{p}(T)$ is finite at any temperature $\left[\omega_{p}(T) \geqslant \sqrt{16 \pi n a^{* 3}} \mathrm{Ry} * / \hbar\right]$; (ii) due to the $\sinh ^{2}\left(\hbar \omega / 2 k_{B} T\right)$ term in the denominator, the integrand in Eq. (2) is significantly large only for $\hbar \omega \leq k_{B} T$, while it decreases exponentially for higher temperatures; and (iii) the plasmon linewidth increases with temperature. Points (i) and (ii) imply that for small temperatures the integrand has already vanished when $\omega \approx \omega_{p}(T)$; on the other side, because of point (iii), for temperatures such that $k_{B} T \lesssim \omega_{p}(T)$, the strength of the plasmon is negligible.

Let us now examine some issues concerning the experimental observation of the SCD.

First of all, it must be clear that the SCD is an intrinsic many-body effect, and, therefore, it is possible to design an experiment to measure it directly and independently of the ordinary diagonal part of the resistivity tensor. Such an experiment has been described in Refs. 4,6.

For different experimental setups, however, it might be important to know how the spin drag resistivity compares to the familiar Drude resistivity $\rho_{D}$. This information is provided in Fig. 6 for doped layers of InAs $\left(m^{*}=0.026 m_{e}, \epsilon\right.$ $=13.6)$ and GaAs $\left(m^{*}=0.067 m_{e}, \epsilon=12\right)$ at two different densities. Since it is customary to express the resistivity of doped semiconductor samples in terms of their mobility, in the upper panel we plot the quantity $\mu_{\uparrow \downarrow}=-1 /$ ne $\rho_{\uparrow \downarrow}$ as a function of temperature. $\mu_{\uparrow \downarrow}$ has the dimensions of a mobility, and its value should be compared to that of the ordinary mobility $\mu_{D}$ of the sample : if, at a given temperature, $\mu_{\uparrow \downarrow}$ $<\mu_{D}$ the spin drag resistivity is larger than the ordinary Drude resistivity. It is evident from this figure that for appropriate but realistic parameters, $\mu_{\uparrow \downarrow}$ and $\mu_{D}$ can be quite comparable. The significant numerical difference between the InAs and GaAs results is primarily due to the larger effective mass and, to a lesser extent, to the smaller dielectric constant of GaAs. The impact of these two parameters on the spin-
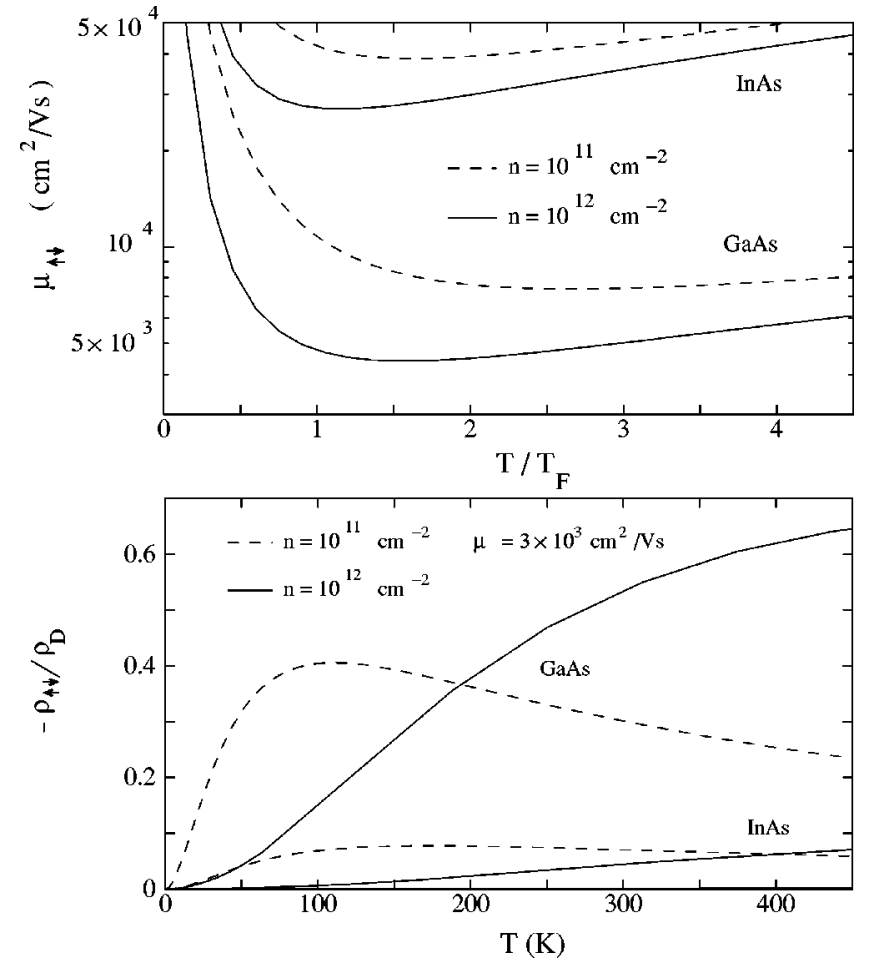

FIG. 6. Upper panel: Spin-drag mobility $\mu_{\uparrow \downarrow} \equiv-1 / \rho_{\uparrow \downarrow} n e$ vs temperature (rescaled by $T_{F}$ ) for $n=10^{11} \mathrm{~cm}^{-2}$ (dashed curve) and $n=10^{12} \mathrm{~cm}^{-2}$ (solid curve). Each couple of curves corresponds to a different material: InAs $\left(m^{*}=0.026 m_{e}, \epsilon=13.6\right)$ and GaAs $\left(m^{*}\right.$ $=0.067 m_{e}, \epsilon=12$ ), as labeled. Lower panel: Ratio $\rho_{\uparrow \downarrow} / \rho_{D}$ as a function of temperature for $n=10^{11} \mathrm{~cm}^{-2}$ (dashed curve) and $n$ $=10^{12} \mathrm{~cm}^{-2} \quad$ (solid curve) and sample mobility $\mu=3$ $\times 10^{3} \mathrm{~cm}^{2} / \mathrm{V} \mathrm{s}$. Each couple of curves corresponds to a different material (InAs and GaAs, as labeled).

drag resistivity is easily understood. Keeping in mind that the SCD is a consequence of Coulomb scattering between different spin populations, a smaller dielectric constant means stronger Coulomb scattering, and a larger effective mass implies a higher density of states available for scattering. Notice that the minimum value of $\mu_{\uparrow \downarrow}$ decreases with increasing density.

In the lower panel of Fig. 6 , we plot the ratio $\rho_{\uparrow \downarrow} / \rho_{D}$ (which is proportional to the sample mobility) versus temperature for a mobility $\mu_{D}=3 \times 10^{3} \mathrm{~cm}^{2} / \mathrm{V} \mathrm{s}$. The same parameters and materials of the upper panel have been chosen. As the figure shows, for reasonable temperatures and realistic parameters, $\rho_{\uparrow \downarrow}$ can be a large fraction of $\rho_{D}$.

A peculiar signature of the two-dimensional SCD, which would be interesting to observe, is the nondegenerate density-independent behavior given by Eq. (18). As shown in the inset of Fig. 4, a sample would display such a behavior at $T \gg T_{F}$, i.e., in the high temperature/low-density regime. Since, for densities of the order of $n=10^{10} \mathrm{~cm}^{-2}, T_{F}$ is as low as $4 \mathrm{~K}$ in GaAs and $11 \mathrm{~K}$ in InAs, this regime should be observable in these materials for temperature considerably lower than room temperature.

Our calculations did not include the finite width of a realistic sample. The response of a quasi-2D sample would be somewhere in between our $3 \mathrm{D}$ and $2 \mathrm{D}$ results, depending on 
how well the idealized two-dimensional approximation is fulfilled. In both the $2 \mathrm{D}$ and $3 \mathrm{D}$ cases however, we see that the ratio $\rho_{\uparrow \downarrow} / \rho_{D}$ remains of the same order of magnitude for the same material and mobility and comparable density (see Fig. 6, lower panel, and Ref. 6, Fig. 2). Moreover, even in the fully three-dimensional case, the nondegenerate regime is only weakly (logarithmically) dependent on the carrier density. The above observations imply that a modest finite-size correction would not modify significantly the relative importance of the predicted effect, or its nondegenerate behavior.

In summary, we have discussed the effect of dimensionality on the spin-transresistivity and shown that in two, as well as in three ${ }^{5,6}$ dimensions, the spin Coulomb drag can be a sizeable effect. We have discussed the differences between Coulomb drag and spin Coulomb drag effects showing how, in the degenerate regime, the different form of the Fourier transform of the Coulomb interaction determines a quantitatively different behavior of the spin transresistivity in comparison to the CD transresistivity. We have also explained why, in general, the spin Coulomb drag is expected to be larger than the ordinary Coulomb drag. Finally we have demonstrated that, in the nondegenerate two-dimensional regime, the spin transresistivity displays a universal densityindependent behavior. Such a behavior could be, in principle, observed experimentally for realistic system parameters.

\section{ACKNOWLEDGMENTS}

We gratefully acknowledge support from NSF Grant No. DMR-0074959.
${ }^{1}$ For a recent review on spin-polarized transport related issues, see S.A. Wolf, D.D. Awschalom, R.A. Buhrman, J.M. Daughton, S. von Molnár, M.L. Roukes, A.Y. Chtchelkanova, and D.M. Treger, Science 294, 1488 (2001) and references therein.

${ }^{2}$ G. Schmidt, D. Ferrand, L.W. Molenkamp, A.T. Filip, and B.J. van Wees, Phys. Rev. B 62, R4790 (2000).

${ }^{3}$ H. Ohno, Science 281, 951 (1998).

${ }^{4}$ I. D'Amico and G. Vignale, Phys. Rev. B 62, 4853 (2000).

${ }^{5}$ I. D'Amico and G. Vignale, Europhys. Lett. 55, 566 (2001).

${ }^{6}$ I. D'Amico and G. Vignale, Phys. Rev. B 65, 085109 (2002).

${ }^{7}$ The issue of spin-flip scattering has been discussed in detail in Ref. 6. There we argued that this contribution is probably very small due to the short range of the spin-flip scattering potential and the weakness of that potential. Here we focus on the Coulomb contribution, which we expect to be larger at experimentally relevant temperatures, and which contains the interesting physics of electronic correlations.

${ }^{8}$ For a recent review of the theoretical and experimental situation on Coulomb drag see A.G. Rojo, J. Phys.: Condens. Matter 11, R31 (1999), and references therein.

${ }^{9}$ The effect of impurity correlation on disorder averaging was examined in Ref. 4 and shown there to vanish in the limit of weak disorder/weak interaction.

${ }^{10}$ In principle, the dielectric "constant" should be frequency dependent due to the vibrational modes of the crystal. In the present treatment, however, the effect of phonons is incorporated only by choosing as dielectric constant $\epsilon$ the average between the static $\left(\epsilon_{0}\right)$ and the "high-frequency" $\left(\epsilon_{\infty}\right)$ dielectric constants.

${ }^{11}$ K. Flensberg and B. Yu-Kuang Hu, Phys. Rev. Lett. 73, 3572 (1994); K. Flensberg and B. Yu-Kuang Hu, Phys. Rev. B 52, 14796 (1995); N.P.R. Hill, J.T. Nicholls, E.H. Linfield, M. Pepper, D.A. Ritchie, G.A.C. Jones, Ben Yu-Kuang Hu, and K. Flensberg, Phys. Rev. Lett. 78, 2204 (1997); H. Noh, S. Zelakiewicz, X.G. Feng, T.J. Gramila, L.N. Pfeiffer, and K.W. West, Phys. Rev. B 58, 12621 (1998).

${ }^{12} \mathrm{An}$ independent calculation of the 2D spin transresistivity has recently been done by K. Flensberg, T.S. Jensen, and N.A. Mortensen, Phys. Rev. B 64, 245308 (2001).

${ }^{13}$ The formal lowest order in the strength of the Coulomb interaction, namely, the second, diverges, due to the infinite range of the interaction. This is remedied by screening the interaction through the inclusion of an infinite series of RPA diagrams, which is expected to be exact in the high-density/weak-coupling limit. RPA should in any case be qualitatively correct at all densities as long as the Landau Fermi-liquid description of the system is valid. As a matter of fact, experiments on the ordinary Coulomb drag (see Ref. 8) were found to qualitatively confirm the main predictions of the RPA even in the low-density regime, which reinforces our expectations that RPA predictions on the spin Coulomb drag will also be qualitatively correct. 